\title{
Development of Effective QCM Biosensors by Cyclopropylamine Plasma Polymerization and Antibody Immobilization using Cross-Linking Reactions
}

\author{
E. Makhneva ${ }^{1,2}$, A. Manakhov ${ }^{1}$, P. Skládal ${ }^{3,4}$, L. Zajíčková ${ }^{1,2 *}$ \\ ${ }^{1}$ RG Plasma Technologies, Central European Institute of Technology (CEITEC), Masaryk \\ University, Kotlářská, 2, Brno 61137, Czech Republic \\ ${ }^{2}$ Department of Physical Electronics, Faculty of Science, Masaryk University, Kotlářská, 2, \\ Brno 61137, Czech Republic \\ ${ }^{3}$ RG Nanobiotechnology, CEITEC, Masaryk University, Kamenice 753/5, Bohunice, Brno \\ 62500, Czech Republic \\ ${ }^{4}$ Department of Biochemistry, Faculty of Science, Masaryk University, Kamenice 753/5, \\ Bohunice, Brno 62500, Czech Republic \\ *Corresponding author : Assoc. Prof. Lenka Zajíčková, \\ Plasma Technologies, CEITEC - Central European Institute of Technology, Masaryk \\ University, Kotlářská, 2, Brno 61137, Czech Republic \\ Tel.+420 54949 8217, Fax. +420 541211 214, lenkaz@ physics.muni.cz
}




\section{Abstract:}

Biosensors have been extensively developed and applied for biomedical and environmental studies. Although there are many different types of biosensing techniques, immobilization of the biorecognition biomolecules onto the sensor surface is always required. Cyclopropylamine pulsed plasma polymerization in radio frequency (RF) capacitive discharges was employed to deposit amine thin films on the gold electrode of quartz crystal microbalance (QCM) biosensors and the monoclonal antibody AL-01, specific to human serum albumin (HSA), was immobilized onto the plasma polymer surface. Two different amine plasma polymers were studied. They had a similar amount of primary amine groups, $1.3-1.5$ at.\%, as determined by chemical derivatization but their stability in water was different. The $1^{\text {st }}$ type, deposited at the floating potential and $120 \mathrm{~Pa}$, exhibited $16 \%$ thickness loss after 24 hours in water whereas the $2^{\text {nd }}$ type deposited at RF electrode and 50 Pa was completely stable (2\% thickness loss). Glutaraldehyde (GA) coupling of AL-01 was employed for both types of the amine films and the performance of QCM immunosensors was evaluated by the immunoassay flow test. A high-stability of the frequency signal was obtained in both the cases but the $2^{\text {nd }}$ type of the film provided low response to HSA. It was explained by its highly cross-linked structure and steric hindrance of active sites. Three different antibody immobilization methods were explored for the $1^{\text {st }}$ type of the film. Stable baseline, selective and high response were recorded for GA and sulfo-SMCC methods. The results confirmed that the presented methodologies for the grafting of biomolecules on the gold are highly efficient and very promising for future use in biosensing. Keywords: plasma polymerization; cyclopropylamine; stable amine-rich films; quartz crystal microbalance; immunosensor; label-free detection. 


\section{Introduction}

Immunosensors with highly sensitive and rapid detection capabilities of various biomolecules are of a great demand in the field of biomedicine and environmental control $[1,2]$. The two mostly employed transducer principles are optical surface plasmon resonance and mass sensitive quartz crystal microbalance (QCM). The QCM is a relatively simple and sensitive device consisting of the AT-cut quartz disc plate covered by evaporated metal electrodes (Au being most common) on both faces. An alternating voltage applied to these electrodes induces shear deformation of the crystal $[3,4]$ and the crystal oscillates with its fundamental resonant frequency $f_{0}$. The first sensor application was developed after Sauerbrey derived the formula relating a change of the oscillation frequency $\Delta f$ to a mass difference $\Delta m$ loaded onto the sensor surface [5]

$$
\Delta f=-\frac{2 f_{0}^{2} \Delta m}{A \sqrt{\rho_{q} \mu_{q}}}
$$

where $A$ the piezoelectrically active area defined by the two gold electrodes, $\rho_{\mathrm{q}}$ the density of quartz $\left(2.648 \mathrm{~g} \mathrm{~cm}^{-3}\right)$ and $\mu_{\mathrm{q}}$ the shear modulus $\left(2.947 \times 10^{11} \mathrm{dyn} \mathrm{cm}^{-2}\right)$.

If a sensitive biorecognition element (antibody) is immobilized onto the surface of one QCM electrode the simple relationship (1) is utilized for the detection of biomolecules (antigens) forming an immunocomplex with antibodies. The QCMs are to be widely used in sensing technology, as evidenced by a large number of publications reporting the use of piezoelectric sensors in various applications such as a cholera toxin diagnostic detection, hepatitis $\mathrm{B}$, hepatitis $\mathrm{C}$, food borne pathogen, influenza $\mathrm{A}$, canine parvovirus, maize chlorotic mottle virus detection [6-13].

The first task encountered in the QCM immunosensor development is the preparation of a coating matrix suitable for the immobilization of antibody on the gold electrode of a quartz crystal. The most popular approaches are based on wet chemical treatments, the 
formation of self-assembled monolayers (SAMs) of alkanethiols and disulfides, or polyethylenimine (PEI) [14,15]. However, these approaches suffer from several drawbacks such as a poor stability, long time preparation, unstable baseline or a high level of noise [4].

As an alternative to above mentioned chemical approaches, the plasma enhanced chemical vapor deposition of thin functional coatings can be employed. It has already been successfully applied to a preparation of plasma polymers containing carboxyl [16-18], amine [19] or anhydride groups [20], deposition of superhydrophobic [21,22] and hard diamond-like carbon [23] thin films. It is known that the essential chemical and thickness stability of the plasma layers can be achieved by tuning the plasma parameters [24,25]. Nakanishi et al. [26] pioneered the plasma polymerization of ethylenediamine (EDA) onto the QCM electrode. It exhibited more stable oscillations in a buffer solution compared to the QCMs coated with the conventional PEI layer. However, the EDA plasma polymers do not exhibit sufficient stability in aqueous environment because 1 min washing in PBS resulted in dissolution of about one third of the film and a significant reduction of $\mathrm{NH}_{2}$ groups (down to 0.4 at.\%) even for the most stable film [27] and, therefore, this approach should be further improved by a deeper investigation of amine plasma polymerization.

Thanks to the reactivity of primary amine groups, amine-rich plasma polymers are extensively employed for biomedical and environmental applications including the immobilization of biomolecules [28,29]. Several monomers can be used for plasma polymerization of amine coatings. Denis et al. suggested to use cyclopropylamine (CPA) as an alternative to commonly used allylamine because CPA was less fragmented in pulsed radio frequency (RF) discharge and yielded higher amount of $\mathrm{NH}_{2}$ groups in the film [30]. Vogelsang et al. succeeded to prepare amine films from CPA and EDA monomers in atmospheric pressure microplasma jet but the films almost completely dissolved after 24 hours in water [31]. Manakhov et al. demonstrated that CPA plasma polymerization in low pressure pulsed RF discharges is efficient for the synthesis of thin polymer films with a high 
content of amine groups (up to 9.2 at.\%) and very good stability in water [32]. In this work, cyclopropylamine plasma polymerization was employed to deposit stable amine-rich thin films on the surfaces of QCM sensors using two different plasma set-ups. In the first group of samples ( $1^{\text {st }}$ type $)$, substrates were placed at the floating potential whereas, in the second group ( $2^{\text {nd }}$ type), the substrates were at DC self-bias. The antibody against human serum albumin (HSA) was attached to the surface via common coupling agent for the immobilization of macromolecules - glutaraldehyde [3,33,34] and both types of plasma polymers were compared. The $1^{\text {st }}$ polymer type, more promising for biosensing application, was tested with two other immobilization approaches besides glutaraldehyde in order to prove the efficiency of plasma polymers for biosensing application. All steps of the bioimmobilizations were controlled by the frequency change of quartz crystals and Fourier transform infrared spectroscopy (FT-IR) to characterizing the surface and layer chemistry.

\section{Experimental}

\subsection{Chemicals and Materials}

Cyclopropylamine (CPA) (98\% purity, used without any further purification), glutaraldehyde (25\% aqueous solution), 4-(trifluoromethyl) benzaldehyde (TFBA) (98\% purity), cysteamine, sulfosuccinimidyl-4-(N-maleimidomethyl)cyclohexane-1-carboxylate (sulfo-SMCC), human serum albumine (HSA) were purchased from Sigma Aldrich. Sodium hydrogenphosphate, sodium dihydrogen phosphate and sodium chloride for the preparation of phosphate buffered saline (PBS) with $\mathrm{pH} 7.4$, ethylenediaminetetraacetate (EDTA) for the preparation of PBS-EDTA buffer with $\mathrm{pH} 7.4$ and sodium periodate were supplied from Penta (Czech Republic). Anti-HSA monoclonal antibody (clone AL01, purified immunoglobulin G, IgG) was obtained from Exbio (Czech Republic). Argon with purity of $99.998 \%$ was supplied by Messer. Double-side polished single crystal silicon (c-Si) wafers (<111〉, N-type phosphorus doped, resistance $0.5 \Omega \mathrm{cm}$ ) from ON-SEMI (Czech Republic) were cut into $10 \mathrm{x}$ 
$15 \mathrm{~mm}$ substrates. Round shape QCMs (AT-cut, $14 \mathrm{~mm}$ in diameter, resonant frequency of 10 MHz) coated by Au with a Cr interlayer were purchased from Krystaly Hradec Králové a.s. (Czech Republic). All the substrates were cleaned by sonication in isopropanol (Penta, 99.8\%) for $10 \mathrm{~min}$.

\subsection{Plasma polymerization}

The plasma polymerization from CPA/Ar gas mixture was performed in capacitively coupled RF discharges (13.56 MHz) of two different plasma reactors described below. All the plasma processes, the surface cleaning and CPA/Ar plasma polymerization, were carried out in square-pulsed mode with the pulse repetition frequency of $500 \mathrm{~Hz}$ and $33 \%$ duty cycle. It corresponded to $660 \mu$ s plasma on-time and $1340 \mu$ s off-time. The leak rate including wall desorption was below $0.1 \mathrm{sccm}$ for all the experiments. The deposition time was tuned to achieve the film thicknesses of 40 and $120 \mathrm{~nm}$ on QCM and Si wafer, respectively. The $40 \mathrm{~nm}$ thick samples were used for immunosensor tests, while $120 \mathrm{~nm}$ thick films were used for FTIR studies.

\subsubsection{Tubular reactor with glass walls ( $1^{\text {st }}$ film type)}

The discharge was ignited in a tubular, horizontally oriented, glass reactor enclosed by aluminium flanges serving as the grounded and RF electrodes. The diameter of the electrodes and their distance were 80 and $185 \mathrm{~mm}$, respectively. The substrates (c-Si and QCMs) were placed on the glass holder positioned in the middle of the tube at the floating potential. The substrate surfaces were cleaned in the Ar discharge for 10 min prior to the deposition. Both, the Ar plasma cleaning and the polymerization, were carried out with the on-time power of 20 $\mathrm{W}$ and at pressure of $120 \mathrm{~Pa}$. The flow rate of Ar was set to $28 \mathrm{sccm}$ whereas the flow rate of CPA vapors was $0.3 \mathrm{sccm}$. A detailed study of the relation between plasma conditions and film properties in the $1^{\text {st }}$ plasma polymerization set-up was reported previously [32] and the 
here utilized deposition conditions were selected as a compromise between a satisfactory $\mathrm{NH}_{2}$ concentration and reasonably low thickness loss.

\subsubsection{Stainless steel vertical plate reactor ( $2^{\text {nd }}$ film type)}

The $2^{\text {nd }}$ plasma polymerization set-up is described in details in the previous paper [35]. The process was carried out in a stainless steel vertical plate reactor. The bottom electrode, $420 \mathrm{~mm}$ in diameter, was capacitively coupled to the RF generator. The gases were fed into the chamber through the grounded upper showerhead electrode, $380 \mathrm{~mm}$ in diameter. The distance between the electrodes was $55 \mathrm{~mm}$. The bottom electrode with substrates was negatively DC self-biased due to an asymmetric coupling. The substrate surfaces were cleaned in the Ar discharge for 10 min prior to the deposition. The surface cleaning and plasma polymerization, were carried out with the on-time power of $100 \mathrm{~W}$ and at pressure of $50 \mathrm{~Pa}$. The flow rate of Ar was set to $28 \mathrm{sccm}$ whereas the flow rate of CPA vapors was 2 sccm. The deposition conditions were selected with the aim to achieve a similar concentration of $\mathrm{NH}_{2}$ groups as in the $1^{\text {st }}$ film type.

\subsection{Preparation of biosensing layers for QCM tests}

Three different procedures were employed for the immobilization of antibodies onto the gold QCM electrode coated with the $1^{\text {st }}$ plasma polymer type as described below. The glutaraldehyde (GA) method was used also for the $2^{\text {nd }}$ film type. After each step, the sensors were thoroughly washed with deionized water, allowed to dry and the resonant frequency was measured using a frequency counter. Standard deviations of the frequency measurements were calculated from the series of three QCM samples characterized for each procedure.

In the first procedure (Figure 1), the surface was activated by the reaction with glutaraldehyde (5\% solution in PBS, $1 \mathrm{~h}$ at room temperature) forming aldehyde groups. 
Then, the AL-01 antibody was covalently bound to these reactive groups $\left(100 \mu \mathrm{g} \cdot \mathrm{mL}^{-1}\right.$ solution in PBS, $18 \mathrm{~h}$ at $\left.4{ }^{\circ} \mathrm{C}\right)[33,34]$.

The second procedure (Figure 2) was based on the reaction of sugar residues of the AL-01 antibody with sodium periodate $\left(\mathrm{NaIO}_{4}, 0.1 \mathrm{M}\right.$ solution in distilled water, $20 \mathrm{~min}$ at room temperature in dark). An oxidation of oligosaccharide chains in the antibody molecule resulted in the creation of reactive aldehyde groups [36]. After the reaction, the sodium sulfite $\left(\mathrm{Na}_{2} \mathrm{SO}_{3}\right)$ was added to provide a double molar excess over the initial amount of the added periodate. The oxidized antibody was purified by dialysis for $1.5 \mathrm{~h}$ and then immediately used for covalent coupling with the amine groups of the CPA plasma polymer $\left(100 \mu \mathrm{g} \cdot \mathrm{mL}^{-1}\right.$ solution in PBS, $18 \mathrm{~h}$ at $\left.4{ }^{\circ} \mathrm{C}\right)$.

For the third method (Figure 3), the disulfide bridges of AL-01 antibody were transformed into the reactive thiol groups [42]. The AL-01 antibody was diluted in PBSEDTA to $2 \mathrm{mg} \cdot \mathrm{mL}^{-1}$ and then reduced $\left(10 \mu \mathrm{L}\right.$ of $60 \mathrm{mg} \cdot \mathrm{mL}^{-1}$ cysteamine were added to 100 $\mu \mathrm{L}$ of antibody solution). The incubation took $90 \mathrm{~min}$ at $37^{\circ} \mathrm{C}$ and the reduced antibodies were purified using the centrifugal microfilter Microcon YM-10 (Merck Millipore, Billerica, MA, USA). The sensor surface coated with plasma polymer was activated by sulfo-SMCC (3 $\mathrm{mg} \cdot \mathrm{mL}^{-1}, 1 \mathrm{~h}$ at room temperature) and finally, the reduced antibody was coupled (100 $\mu \mathrm{g} \cdot \mathrm{mL}^{-1}, 18 \mathrm{~h}$ at $4{ }^{\circ} \mathrm{C}$ ) through thiol-groups and maleimide-fragment interaction.

\subsection{QCM measurements}

The immunoaffinity experiments (Figure 4) involved a binding interaction between the AL-01 antibody immobilized on the QCM electrode and the solution of HSA as antigen (Ag). The piezoelectric crystals were placed in a flow-through cell (designed and constructed by Karel Lacina). The measurements were performed using the QCM Analyzer (KEVA, Brno, Czech Republic) that served as both, the oscillator and the frequency counter. The flow of solutions was driven by the milliGAT pump (Global FIA, Fox Island, WA, USA) and a 
selection valve (Valco Instruments, Houston, TX, USA). The whole system was controlled via the in-house developed software LabTools that allowed fully automated operation. PBS was used as a running buffer with a flow rate of $40 \mu \mathrm{L} \cdot \mathrm{min}^{-1}$. Intially, the free reactive groups were deactivated using bovine serum albumine (BSA, $200 \mu \mathrm{g} / \mathrm{ml}, 10 \mathrm{~min}$ at room temperature). After baseline stabilization the samples of HSA were injected for $10 \mathrm{~min}$ followed by 10 min dissociation phase in PBS. Changes of the mass on the gold electrode after different steps were calculated from the QCM frequency measured in a dry state using Eq. (1).

\subsection{FT-IR analysis}

Fourier transformed infrared (FT-IR) spectra were obtained on the films deposited on the IR transparent c-Si substrates in the transmission mode using the Bruker Vertex 80v spectrophotometer. The measurements were performed with a parallel beam transmittance accessory in the spectral range from 370 to $7500 \mathrm{~cm}^{-1}$. The data were collected at pressure of 2.5 mbar with the resolution of $4 \mathrm{~cm}^{-1}$ and 500 scans. The transmittance of the films on $\mathrm{Si}$ substrate was divided by the transmittance of bare Si substrate for the thin film analysis. The FT-IR spectra are shown only in the range from 1490 to $3650 \mathrm{~cm}^{-1}$ because no significant absorption peaks belonging to the films were identified outside this range and the identification of weak absorption peaks below $1490 \mathrm{~cm}^{-1}$ was difficult due to the strong absorption peaks in c-Si that could not be reliably subtracted.

\subsection{XPS analysis and chemical derivatization.}

X-ray photoelectron spectroscopy (XPS) analysis of CPA films deposited onto c-Si was performed in a Kratos Axis-Ultra DLD system using monochromatic Al-Ka radiation (Xray power $150 \mathrm{~W}$ ) in normal emission geometry. The pass energy was set to 20 and $160 \mathrm{eV}$ for detailed regions and wide spectra, respectively. The detailed spectra was acquired in 0.1 
$\mathrm{eV}$ energy steps with total acquisition time ranging from 600 to $1800 \mathrm{~s}$ to ensure good signal to noise ratio. The charge neutralizer was used to compensate the charging of insulating sample during the analysis.

The quantification of the primary amine groups was performed by chemical derivatization combined with XPS, CD-XPS. The well known derivatization using TFBA was employed as shown in Figure 5 [37]. Due to high sensitivity of TFBA to moisture and oxygen, the reaction was carried out in an Ar atmosphere. The CPA plasma layers and clean Si wafer were placed in the glovebox immediately after the preparation. Then, glovebox chamber was pumped down with membrane pump and filled with Ar. The procedure was repeated 3 times to avoid the residue of oxygen and moisture. Each sample was placed on the top of the glass beads inside the $100 \mathrm{ml}$ flask and then $0.1 \mathrm{ml}$ of TFBA was dropped in such way to avoid the contact of liquid with the sample surface. Then flask was closed and the reaction was proceeding for 90 minutes. The density of primary amines $\left[\mathrm{NH}_{2}\right]$ expressed in at.\% was calculated from the fluorine and carbon atomic concentrations measured by XPS similarly as reported in literature $[37,38]$.

\section{Results and discussion}

\subsection{Chemical composition and properties of CPA plasma polymers}

In our recent studies it was shown that pulsed plasma polymerization of CPA can be employed to produce the amine-rich films sufficiently resistant to water [32,35]. It was proved by ellipsometric measurements that after immersion 24 hours in water the $1^{\text {st }}$ film type is water-stable up to $84 \%$ of the original thickness [32] whereas the $2^{\text {nd }}$ film type exhibits the thickness loss of only $2 \%$ [35]. Further prolongation of the water immersion did not change the film thickness. Therefore, it is concluded that after washing of unstable oligomers, both the plasma polymer coatings exhibit high level of stability.

A decrease of the QCM original frequency in the first step, plasma polymerization (PP), was related to a mass of deposited plasma polymer film. By taking into account the 
thickness of the CPA films and the area of the QCM electrodes, it was found that the densities of the plasma polymers are equal to 1.30 and $1.32 \mathrm{~g} / \mathrm{cm}^{3}$ for the $1^{\text {st }}$ and $2^{\text {nd }}$ film types, respectively.

Both types of the CPA films had similar atomic composition [32, 35]. According to the XPS analyses of $40 \mathrm{~nm}$ thick films of the $1^{\text {st }}$ and $2^{\text {nd }}$ type, the $\mathrm{C}: \mathrm{N}: \mathrm{O}$ ratios were 77:20:3 and 78:18:4, respectively. It was proved that the atomic concentration of elements for the 120 $\mathrm{nm}$ thick films varied only within the error of XPS measurements (1-2 at.\%). Since the immobilization strategies rely on the reactivity of primary amines it was necessary to evaluate also $\mathrm{NH}_{2}$ density. The curve fitting of $\mathrm{N} 1$ s signal could not provide this information because binding energies of primary and secondary amines are almost the same. Although there are many debates regarding the selectivity of CD-XPS using TFBA it can provide a reasonable density of reactive groups available for the coupling of antibodies using $\mathrm{GA}$ or $\mathrm{NaIO}_{4}$ method because the immobilization mechanism is based on a similar aldehyde condensation reaction as employed in the TFBA derivatization procedure. The concentration of primary amine groups determined by CD-XPS were 1.3 and 1.5 at.\% for the $1^{\text {st }}$ and $2^{\text {nd }}$ film types, respectively. Additionally, FT-IR analyses revealed the presence of primary amines by $\mathrm{NH}_{2}$ scissoring absorption peak at $1640 \mathrm{~cm}^{-1}$, primary and secondary amines by stretching around $3360 \mathrm{~cm}^{-1}$, hydrocarbons by $\mathrm{CH}_{2}$ and $\mathrm{CH}_{3}$ stretching in the range $2870-2930 \mathrm{~cm}^{-1}$ and nitriles/isonitriles $\left(-\mathrm{C} \equiv \mathrm{N}\right.$ and $\mathrm{C} \equiv \mathrm{N}^{+}-/-\mathrm{N}=\mathrm{C}=\mathrm{N}-$ at $\left.2190 \mathrm{~cm}^{-1}\right)$.

\subsection{Comparison of plasma polymers concerning thein performance in biosensing.}

In the second step of immobilization utilizing GA, the gold electrodes coated by the CPA plasma polymers were activated by the reaction with GA (PP-GA samples). The robust GA immobilization method was tested for both types of the films in order to decide about the perspectives in a replacement of SAMs in QCM immunosensors. Despite of a random mechanism of the GA immobilization, it has been shown before that there are no conformational changes in the antibody active sites [38].

All the procedure steps, i.e. PP, PP-GA and immobilization of antibody (PP-GA-Ab), were followed by the measurements of QCM frequency (Table 1) and investigation of chemistry by FT-IR (Figure 6). The PP_GA step was accompanied by a slight addition of the 
film mass and chemical changes observable by FT-IR. The decreased intensity of the peaks assigned to $\mathrm{NH}_{2}$-group was accompanied by an increase of $\mathrm{CH}_{2}$ stretching and the appearance of the carbonyl $\mathrm{C}=\mathrm{O}$ stretching at $1709 \mathrm{~cm}^{-1}$ from the immobilized glutaraldehyde. Due to an overlap of the carbonyl stretching of aldehyde, ester and carboxyl groups around $1700 \mathrm{~cm}^{-1}$, different carbonyl environments could not be distinguished $[39,40]$. However, it is natural to assume that the carbonyls in the FT-IR spectra after the PP-GA-Ab step are related to the carboxyl groups from the antibody protein nature. Additionally, the increase of the $\mathrm{CH}_{2}$ stretching peak and the appearance of amide bonds from antibody molecule (NH deformation at $1570 \mathrm{~cm}^{-1}$ and $\mathrm{C}=\mathrm{O}$ stretching at $\sim 1650 \mathrm{~cm}^{-1}$ ) confirmed the grafting of the antibody. Comparing the peak intensity changes during transformations, it can be concluded that the yield of the reactions was considerably lower for the $2^{\text {nd }}$ than $1^{\text {st }}$ film type.

The sensors with the immobilized AL-01 antibody were used for the detection of the complementary HSA-antigen. It is known that this pair provides a quite reliable immunocomplex under different conditions and assay formats [41]. For both the samples the baseline was well established because the signal in the flowing PBS was stable for hours (Figure 7 shows it only for $30 \mathrm{~min}$ in order to have a concise $\mathrm{x}$-axis). The mean baseline signal calculated from data in flowing PBS during 90 min was $-0.12 \pm 0.73 \mathrm{~Hz}$ for the $1^{\text {st }}$ film type and $-0.11 \pm 0.89 \mathrm{~Hz}$ for the $2^{\text {nd }}$ film type. A decreased frequency, induced by the formation of the immunocomplex, was clearly observed after the injection of HSA/PBS. The delay in the frequency response was caused by a delay in the injection system.

The high stability level of binding interaction curves was observed for both types of plasma polymer films. However, the response of the sensors with the $2^{\text {nd }}$ film type was much lower compared to the response of the sensors based on the $1^{\text {st }}$ film type. A sharp decrease of the QCMs resonant frequency by $40 \mathrm{~Hz}$ achieved during the reaction with $10 \mu \mathrm{g} / \mathrm{ml}$ solution of HSA in case of $1^{\text {st }}$ film type is typical for the GA method [42]. According to the literature related to conventional SAMs $[43,44]$, stability level of the developed immunosensors was 
higher. Because of the better response the $1^{\text {st }}$ type of the plasma polymer film was chosen for further investigations.

\subsection{Comparison of three antibody immobilization procedures.}

Three different immobilization procedures (GA, sodium periodate and sulfo-SMCC) were applied and studied for the $1^{\text {st }}$ plasma polymer film type selected in the previous section because of its better efficiency in biosensing.

According to Figure 2 for the periodate method, the antibody was at first activated by periodate-oxidation to contain reactive aldehyde residues. As oligosaccharide residues are typically located in the $F_{c}$ domain of immunoglobulins it should be beneficial for the expected orientation of immobilized antibody, contrary to the GA method leading to a coupling to both $\mathrm{F}_{\mathrm{ab}}$ and $\mathrm{F}_{\mathrm{c}}$ domains. The oxidized antibody was covalently bound to the amine-rich plasma polymer deposited onto the QCM Au electrode surface.

The disulfide bridges of AL-01 antibody were transformed into reactive thiol groups in case of the third procedure (Figure 3). A partial reduction is a commonly used reaction for the activation of antibodies because it does not affect their functionality - no decrease of complement activation efficiency [45] and absence of global conformational changes of $\mathrm{Ab}$ molecule [46]. Sulfo-SMCC reagent was linked to the substrates coated with CPA-plasma polymer and then the reduced antibody was covalently bound through thiol-groups and maleimide-fragment coupling. For this approach, it is expected that the active cites of the antibody are more accessible for an antigen binding. However, it is a time and cost consuming procedure compared to the GA and periodate methods.

The control of the immobilization of antibody was carried out by measuring the resonant frequency changes of quartz crystals after the PP-xx-Ab step (where $\mathrm{xx}$ is GA, $\mathrm{NaIO} 4$ or sulfo-SMCC). The frequency changes resulting in small mass increases were observed in all the cases (Table 2). 
The kinetics of the reaction between immobilized antibody and the solution of HSAantigen is shown in Figure 8. Although it was proposed that for the sodium periodate method the conjugation is taking place through the oligosaccharide chains without compromising the antibody activity, a high level of noise and unstable baseline (during the measurement up to 30 minutes after binding interaction) were recorded. For the sulfo-SMCC method, a stable baseline and the frequency decrease of $16 \mathrm{~Hz}$, typical for this method [41], were recorded. The kinetic curves obtained from GA and sulfo-SMCC methods had similar shape with a sharp drop and stable baseline after the binding interaction, which is typical to observe when the sensor is effective [47]. Usually the GA-method is applied for analysis of probes with high concentration of antigen, while the sulfo-SMCC is usually used for small concentrations, because it is a very accurate and sensitive method [41].

All the developed immunosensors were tested at different concentrations of solution of HSA-antigen solutions: $10 \mu \mathrm{g} / \mathrm{ml}, 20 \mu \mathrm{g} / \mathrm{ml}, 50 \mu \mathrm{g} / \mathrm{ml}$ and $100 \mu \mathrm{g} / \mathrm{ml}$. For the GA and sulfoSMCC methods, the stable response was achieved for each concentration of the HSA-antigen indicating negligible dissociation of the immunocomplex. Typically, the dependence of frequency change on the antigen concentration exhibits a saturation character and, therefore, the linearization is achieved by plotting the concentration on the $\mathrm{x}$-axis in log-scale [41]. Figure 9 provides calibration curves for the QCM detection of HSA-antigen. The error bars correspond to the standard deviations. In the range of used concentrations, the GA and sulfoSMCC immobilization methods exhibited very good linear response using the log-scale of xaxis. The linear correlation coefficients were 0.995 and 0.999 for GA and sulfo-SMCC methods, respectively. The limit of detection for albumin estimated for the best immunosensor, i.e. antibody immobilized on the $1^{\text {st }}$ plasma polymer film using the GA method, was around $5 \mu \mathrm{g} / \mathrm{ml}$ taking into account the mean level of noise of $1 \mathrm{~Hz}$ and the signal-to-noise ratio of 3. 


\section{Conclusions}

Pulsed plasma polymerization of CPA is an effective method for the preparation of amine-containing films that are proposed as a replacement of SAMs on the gold electrode of QCM sensors. Two types of amine-rich polymer films were deposited in the CCP discharge at different potential and pressure. They had a similar amount of primary amine groups, $1.3-$ 1.5 at.\%, as determined by chemical derivatization, but the film stability in water was different. The $1^{\text {st }}$ type of amine polymer, deposited at the floating potential (120 Pa), exhibited $16 \%$ thickness loss after 24 hours in water whereas the $2^{\text {nd }}$ type of polymer deposited at RF electrode $(50 \mathrm{~Pa})$ was completely stable ( $2 \%$ thickness loss). Further prolongation of the water immersion did not change the film thickness. It led to the conclusion that after washing of unstable oligomers, both the plasma polymer coatings exhibit high level of stability.

The GA coupling of AL-01 was employed for both types of the amine films and the performance of QCM immunosensors was evaluated by the immunoassay flow test probing the interaction of immobilized AL-01 and HSA as a model. Both types of QCM immunosensors exhibited high-stability of the signal but the $2^{\text {nd }}$ type of the film provided low response to HSA. The lower level of response can be explained by highly cross-linked structure of this polymer and steric hindrance of active sites. Three different antibody immobilization approaches were explored for the $1^{\text {st }}$ type of the film that showed promising result for immunosensing. Stable baseline, selective and high response were recorded for the GA and sulfo-SMCC methods. The results confirmed that the presented methodologies for the grafting of biomolecules on the gold surfaces have great potential for biosensing applications

\section{Acknowledgements}

The research was also supported by the project "CEITEC — Central European Institute of Technology" (CZ.1.05/1.1.00/02.0068) from the European Regional Development Fund and the SCOPES project IZ73Z0_152661 funded by Swiss National Science Foundation. The 
authors are grateful to Dr. Lukáš Kalina from Brno University of Technology for carrying the XPS analyses. 


\section{References}

[1] M. Cooper, V.T. Singleton, A survey of the 2001 to 2005 quartz crystal microbalance biosensor literature: applications of acoustic physics to the analysis of biomolecular interactions, J. Mol. Recognit. 20 (2007) 154-184.

[2] M. Malmqvist, BIACORE: an affinity biosensor system for characterisation of biomolecular interactions, Biochem. Soc. Trans. 27 (1998) 335-340.

[3] S. Babacan, P. Pivarnik, S. Letcher, A. Rand, Evaluation of antibody immobilisation methods for piezoelectric biosensor application, Biosens. Bioelectron. 15 (2000) 615621.

[4] P. Peluso, D. Wilson, D. Do, H. Tran, M. Venkatasubbaiah, D. Quincy, B. Heidecker,K. Poindexter, N. Tolani, M. Phelan, K. Witte, L.S. Jung, P. Wagner, S. Nock, Optimizing antibody immobilization strategies for the construction of protein microarrays, Anal. Biochem. 312 (2003) 113-124.

[5] G. Sauerbrey, Verwendung von schwingquarzen zur wägung dünner schichten und zur mikrowägung, Z. Phys. 1959, pp. 155, 206-222.

[6] P. Skládal, Piezoelectric Quartz Crystal Sensors Applied for Bioanalytical Assays and Characterization of Affinity Interactions, J.Braz.Chem.Soc. 14 (2003) 491-502.

[7] J. Chen, K. Futami, D.Petillo, J. Peng, P. Wang, J. Knol, Y. Li, Deficiency of FLCN in mouse kidney led to development of polycystic kidneys and renal neoplasia, PLoS ONE 310 (2008) e3581.

[8] J. Chen, E. Milliman, I. Goulet, C. Jackson, J. Vollbracht, M. Yu, Protein arginine methylation facilitates cotranscriptional recruitment of pre-mRNA splicing factors, Mol. Cell. Biol. 3021 (2010) 5245-5256.

[9] J. Yao, A. Phan, D. Chang, R. Wolff, K. Hess, S. Gupta, Efficacy of RAD001 (everolimus) and octreotide LAR in advanced low- to intermediate-grade neuroendocrine tumors: results of a phase II study, J. Clin. Oncol. 26 (2008) 4311-8.

[10] T. Owen, R. Al-Kaysi, C. Bardeen, Q. Cheng, Microgravimetric immunosensor for direct detectionof aerosolized influenza A virus particles, Sensors and Actuators B 126 (2007) 691-699.

[11] F. Dultsev, A. Tronin, Rapid sensing of hepatitis B virus using QCM in the thickness shear mode, Sensors and Actuators, B 216, 1 (2015), 1-5

[12] Y. Kim, S. Lim, S. Choi, I.-S. Cho, E.-H. Park, D.-J. An, A novel assay for detecting canine parvovirus using a quartz crystal microbalance biosensor, Journal of Virological Methods 219, (2015), 23-27. 
[13] X. Huang, J. Xu, H.-F. Ji, G. Li, H. Chen, Quartz crystal microbalance based biosensor for rapid and sensitive detection of maize chlorotic mottle virus, Analytical Methods 6 , 13, (2014), 4530-4536.

[14] Z. Fohlerova, P. Skladal, J. Turanek, Adhesion of eukaryotic cell lines on the gold surface modified with extracellular matrix proteins monitored by the piezoelectric sensor, Biosens. Bioelectron. 22 (2007) 1896-1901.

[15] K. Marx, Quartz Crystal Microbalance: A Useful Tool for Studying Thin Polymer Films and Complex Biomolecular Systems at the Solution-Surface Interface, Biomacromolecules 4 (2003) 1099-1120.

[16] M. Donegan, D.P. Dowling, Protein adhesion on water stable atmospheric plasma deposited acrylic acid coatings, Surf. Coat. Technol. 234 (2013) 53-59.

[17] Y.P. Li, Z.C. Zhang, W. Shi, M.K. Lei, Adhesion enhancement of polyethylene modified by capacitively coupled radio frequency plasma polymerization of ethanol, Surf. Coat. Technol. (2014) 259.

[18] M. Moreno-Couranjou, A. Manakhov, N.D. Boscher, J.-J. Pireaux, P. Choquet, A Novel Dry Chemical Path Way for Diene and Dienophile Surface Functionalization toward Thermally Responsive Metal-Polymer Adhesion, ACS Appl. Mater. Interfaces 5 (2013) 8446-8456.

[19] M. Crespin, N. Moreau, B. Masereel, O. Feron, B. Gallez, T. Vander Borght, C.Michiels, S. Lucas, J. Mater, Surface properties and cell adhesion onto allylamine-plasma and amine-plasma coated glass coverslips, Sci. Mater. Med. 22 (2011) 671-682.

[20] A. Manakhov, M. Moreno-Couranjou, P. Choquet, N.D. Boscher, J.-J. Pireaux, Atmospheric Pressure Pulsed Plasma Copolymerisation of Maleic Anhydride and Vinyltrimethoxysilane: Influence of Electrical Parameters on Chemistry, Morphology and Deposition Rate of the Coatings, Surf. Coat. Technol. (2011) 205.

[21] E. Kedroňová, L. Zajíčková, D. Hegemann, M. Klíma, M. Michlíček, A. Manakhov, Plasma Enhanced CVD of Organosilicon Thin Films on Electrospun Polymer Nanofibers, Plasma Process. Polym. (2015) doi:10.1002/ppap.201400235.

[22] R. Múgica-vidal, F. Alba-elías, E. Sainz-garcía, J. Ordieres-meré, Atmospheric plasmapolymerization of hydrophobic and wear-resistant coatings on glass substrates, Surf. Coat. Technol. 259 (2014) 374-385.

[23] A. Stoica, A. Manakhov, J. Polčák, P. Ondračka, V. Buršíková, R. Zajíčková, Cell proliferation on modified DLC thin films prepared by plasma enhanced chemical vapor deposition Cell proliferation on modified DLC thin films prepared by plasma enhanced chemical vapor deposition, Biointerphases. (2015) 029520 doi:10.1116/1.4920978.

[24] D. Hegemann, B. Hanselmann, S. Guimond, G. Fortunato, M.-N. Giraud, A.G. Guex, Considering the degradation effects of amino-functional plasma polymer coatings for biomedical application, Surf. Coat. Technol. 255 (2014) 90-95.

[25] A. Manakhov, M. Moreno-Couranjou, N.D. Boscher, V. Roge, P. Choquet, J.-J.Pireaux, Atmospheric Pressure Pulsed Plasma Copolymerisation of Maleic Anhydride and 
Vinyltrimethoxysilane: Influence of Electrical Parameters on Chemistry, Morphology and Deposition Rate of the Coatings, Plasma Process. Polym. 9 (2012) 435-445.

[26] K. Nakanishi, H. Muguruma, I. Karube, A novel method of immobilizing antibodies on a quartz crystal microbalance using plasma-polymerized films for immunosensors, Anal. Chem. 68 (1996) 1695-1700.

[27] C. Daunton, L. Smith, J. Whittle, R. Short, D. Steele, Plasma Parameter Aspects in the Fabrication of Stable Amine Functionalized Plasma Polymer Films, Plasma Process. Polym. (2015) doi: 10.1002/ppap.201400215.

[28] K. Siow, L. Britcher, S. Kumar, H.J. Griesser, Plasma Methods for the Generation of Chemically Reactive Surfaces for Biomolecule Immobilization and Cell Colonization - A Review, Plasma Process. Polym. 3 (2006) 392-418.

[29] H. Muguruma, Plasma-polymerized films for biosensors II, Trends Anal. Chem. 26 (2007) 433-443.

[30] L. Denis, P. Marsal, Y. Olivier, T. Godfroid, R. Lazzaroni, M. Hecq, J. Cornil, R. Snyders, Deposition of Functional Organic Thin Films by Pulsed Plasma Polymerization: A Joint Theoretical and Experimental Study, Plasma Process. Polym. 7 (2009) 172-181.

[31] A. Vogelsang, A. Ohl, R. Foest, K. Schroder, K.-D. Weltmann, Deposition of Thin Films from Amino Group Containing Precursors with an Atmospheric Pressure Microplasma Jet, Plasma Process. Polym. 8 (2011) 77-84.

[32] A. Manakhov, L. Zajíčková, M. Eliáš, J. Čechal, J. Polčák, J. Hnilica, Š. Bittnerová, D. Nečas, Optimization of Cyclopropylamine Plasma Polymerization Towards Enhanced Layer Stability in Contact with Water, Plasma Process. Polym. 11 (2014) 532-544.

[33] V. Sai, S. Mahajan, A. Contractor, S. Mukherji, Immobilization of antibodies on polyaniline films and its application in a piezoelectric immunosensor, Anal. Chem. 78 (2006) 8368-8373.

[34] T. Huy, N.Hanh, P.Chung, D.Anh, P.Nga, M.Tuan, Characterization of immobilization methods of antiviral antibodies in serum for electrochemical biosensors, Appl. Surf. Science 257 (2011) 7090-7095

[35] A. Manakhov, D. Nečas, J. Čechal, D. Pavliňak, M. Eliaš, L. Zajičkova, Deposition of stable amine coating onto polycaprolactone nanofibers by low pressure cyclopropylamine plasma polymerization, Thin Solid Films 581 (2015) 7-13.

[36] G. Shen, X. Zhang, Y. Shen, S. Zhang, L. Fang, One-step immobilization of antibodies for a-1-fetoprotein immunosensor based on dialdehyde cellulose/ionic liquid composite, Anal. Biochem. 471 (2015) 38-43.

[37] P. Favia, M.V. Stendardo, R. d'Agostino, Selective grafting of amine groups on polyethylene by means of $\mathrm{NH}_{3}-\mathrm{H}_{2} \mathrm{RF}$ glow discharges, Plasmas Polym. 1 (1996) 91112. 
[38] A. Manakhov, P. Skládal, D. Nečas, J. Čechal, J. Polčák, M. Eliáš, L. Zajíčková, Cyclopropylamine plasma polymers deposited onto quartz crystal microbalance for biosensing application, Physica Stat. Solid. (a) 21112 (2014) 2801-2808.

[39] G. Socrates, Infrared and Raman characteristic group frequencies. Tables and charts, Wileys\&Sons: Chimester, 2004.

[40] D. W. Mayo, F. Miller, R. H. Interpretation of infrared and Raman spectra, Wileys\&Sons: New Jersey, 2004.

[41] Z. Farka , D. Kovár, P. Skládal, Rapid Detection of Microorganisms Based on Active and Passive Modes of QCM, Sensors 15 (2015) 79-92.

[42] I. Navratilova, P. Skladal, V. Viklicky, Development of piezoelectric immunosensors for measurement of albuminuria, Talanta 55 (2001) 831-839.

[43] S.-F.Chou, W.-L. Hsu, J.-M. Hwang, C.-Y. Chen, Determination of alpha-fetoprotein in human serum by a quartz crystal microbalance-based immunosensor, Clinical Chemistry 486 (2002) 913-918.

[44] K. Yokoyama, K. Ikebukuro, E. Tamiya, I. Karube, N, Ichiki, Y. Arikawa, Highly sensitive quartz crystal immunosensors for multisample detection of herbicides, Analytica Chimica Acta 3042 (1995) 139-145.

[45] D. Burton, Immunoglobulin G: functional sites, Mol. Immunol. 22 (1985) 161-206.

[46] G. Seegan, C. Smith, V. Schumaker, Changes in quaternary structure of IgG upon reduction of the interheavy-chain disulfide bond, Proc. Natl. Acad. Sci. USA. 76 (1979) 907-911.

[47] K. Vashist, P. Vashist, Recent Advances in Quartz Crystal Microbalance-Based Sensors, Journal of Sensors (2011) 1-13 doi:10.1155/2011/571405. 
Figure captions

Fig. 1 Immobilization of antibody using the glutaraldehyde method.

Fig. 2 Immobilization of antibody using the sodium periodate method.

Fig. 3 Immobilization of antibody using the sulfo-SMCC method.

Fig. 4 Antibody (AL-01) - antigen (HSA) binding interactions for different immobilization approaches.

Fig. 5 The scheme of the primary amines derivatization

Fig. 6 FT-IR spectra at different antibody immobilization steps: $a-1^{\text {st }}$ film type, $b-2^{\text {nd }}$ film type.

Fig. 7 Signal traces from the immunointeraction between immobilized AL-01 antibody and 10 $\mu \mathrm{g} / \mathrm{ml}$ solution of HSA-antigen: $\mathrm{a}-1^{\text {st }}, \mathrm{b}-2^{\text {nd }}$ type of PP film on the surface of QCM. The curve $\mathrm{c}$ shows stability in pure PBS of the less stable $1^{\text {st }}$ film type with immobilized AL-01. Fig. 8 Signal traces from the interactions, $c(\mathrm{HSA})=10 \mu \mathrm{g} / \mathrm{mL}$, coupling of Ab carried out using: a - GA, b - $\mathrm{NaIO}_{4}$ and c - sulfo-SMCC-methods.

Fig. 9 Calibration curves for the HSA antigen obtained for the compared antibody immobilization methods. 
Table 1. Changes of the QCM frequency and corresponding mass differences at different steps of the GA immobilization procedure, i.e. after the deposition of plasma polymer (PP), after the activation of plasma polymer with GA (PP-GA) and after the immobilization of antibody (PP-GA-Ab).

\begin{tabular}{|l|l|l|l|l|l|l|}
\hline $\begin{array}{l}\text { Immobilization } \\
\text { step }\end{array}$ & PP & \multicolumn{2}{|l|}{ PP-GA } & \multicolumn{2}{|l|}{ PP-GA-Ab } \\
\hline & $\Delta f(\mathrm{~Hz})$ & $\Delta m(\mathrm{ng})$ & $\Delta f(\mathrm{~Hz})$ & $\Delta m(\mathrm{ng})$ & $\Delta f(\mathrm{~Hz})$ & $\Delta m(\mathrm{ng})$ \\
\hline $1^{\text {st }}$ film type & $-1661 \pm 24$ & $+1442 \pm 21$ & $-152 \pm 10$ & $+132 \pm 9$ & $-104 \pm 10$ & $+90 \pm 9$ \\
\hline $2^{\text {nd }}$ film type & $-1316 \pm 23$ & $+1142 \pm 20$ & $-97 \pm 7$ & $+84 \pm 6$ & $-89 \pm 5$ & $+77 \pm 4$ \\
\hline
\end{tabular}


Table 2. QCM frequency and mass changes after immobilization of antibody (PP-xx-Ab) and the binding interactions with HSA (PP-xx-Ab-HSA) for three different procedures. This study was performed only for the $1^{\text {st }}$ film type.

\begin{tabular}{|c|c|c|c|c|}
\hline Coupling method (xx) & \multicolumn{2}{|c|}{$\mathrm{PP}-\mathrm{xx}-\mathrm{Ab}$} & $\mathrm{PP}-\mathrm{xx}-\mathrm{Ab}-\mathrm{HSA}$ \\
\hline & $\Delta f(\mathrm{~Hz})$ & $\Delta m(\mathrm{ng})$ & $\Delta f(\mathrm{~Hz})$ & $\Delta m(\mathrm{ng})$ \\
\hline $\mathrm{GA}$ & $-104 \pm 10$ & $+90 \pm 9$ & $-40 \pm 6$ & $+35 \pm 5$ \\
\hline $\mathrm{NaIO}_{4}$ & $-207 \pm 11$ & $+180 \pm 10$ & $-28 \pm 6$ & $+25 \pm 5$ \\
\hline sulfo-SMCC & $-157 \pm 7$ & $+136 \pm 6$ & $-16 \pm 3$ & $+14 \pm 2$ \\
\hline
\end{tabular}




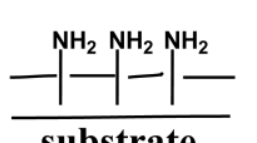

-

PP
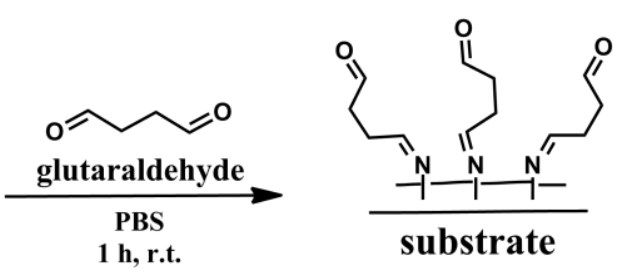

PP-GA
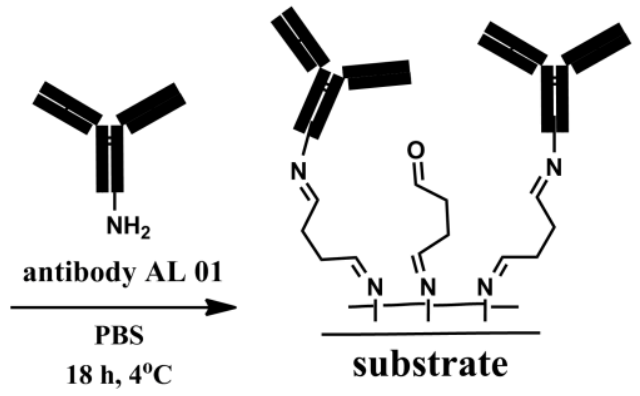

PP-GA-Ab 


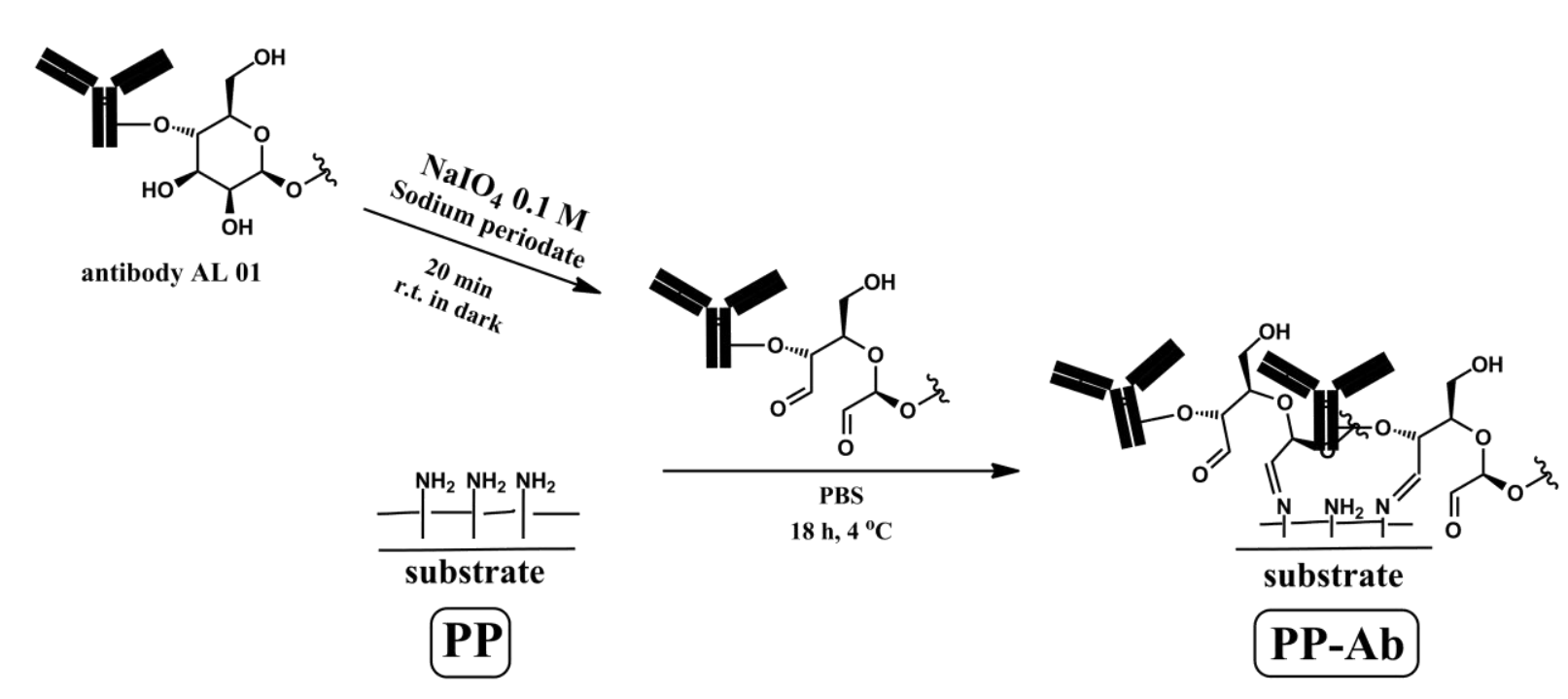




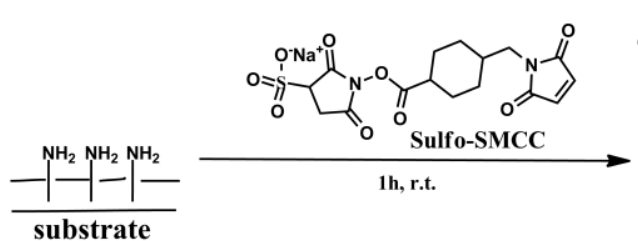

PP

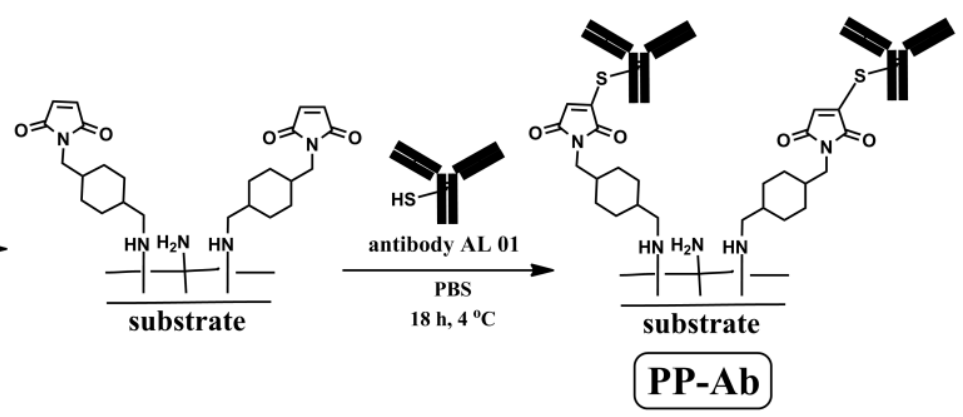




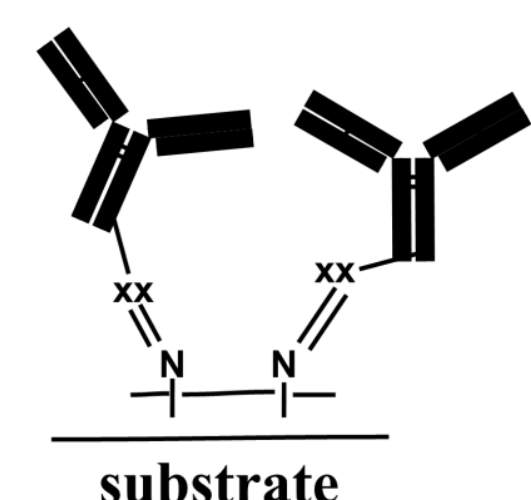

PP-xx-Ab

$\mathrm{xx}: \widehat{\sim}_{\mathrm{N}^{\prime}}$ GA-method

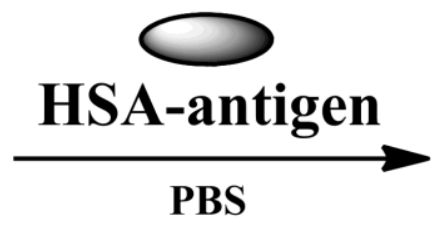

$10 \mathrm{~min}$, r.t.

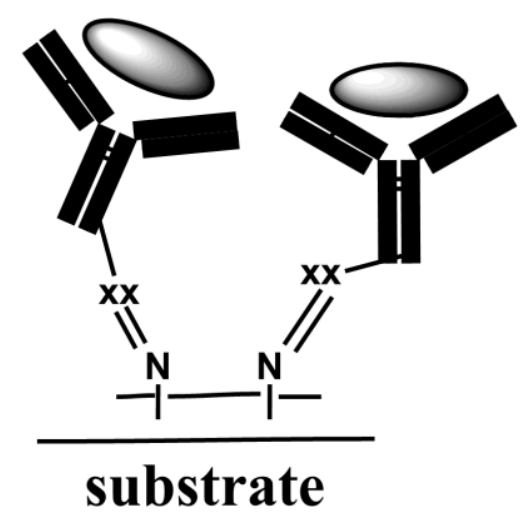

PP-xx-Ab-Ag

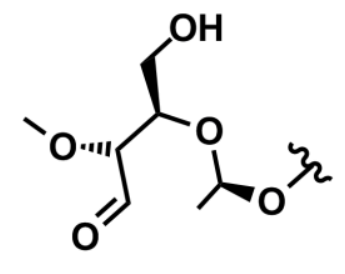

$\mathrm{NaIO}_{4}$-method<smiles>CSC1=CC(=O)N(CC2CCC(C)CC2)C1=O</smiles>

sulfo-SMCC-method 
$\frac{-||^{\mathrm{NH}_{2}} \stackrel{\mathrm{NH}_{2}}{\mathrm{NH}_{2}}-\mid-}{\text { substrate }}$
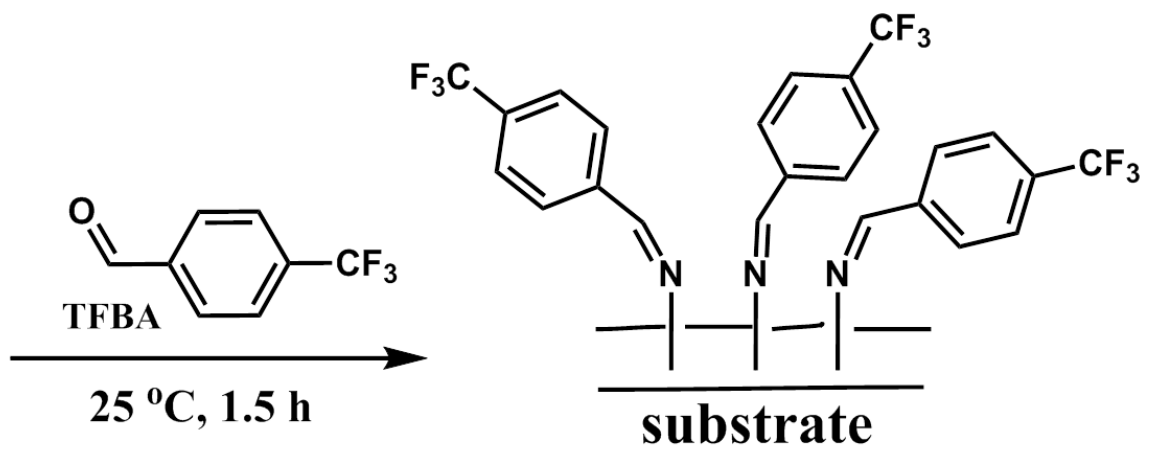



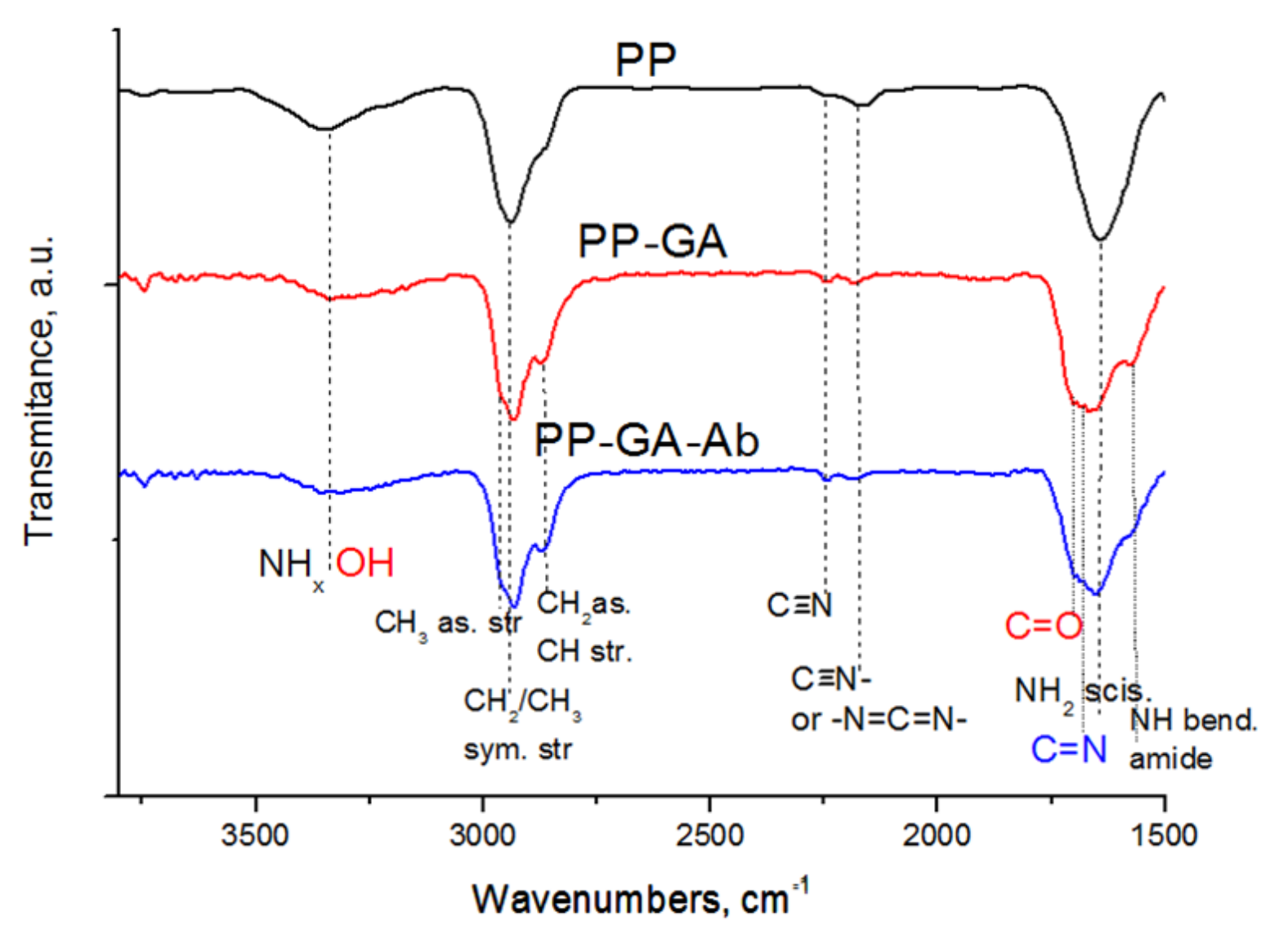




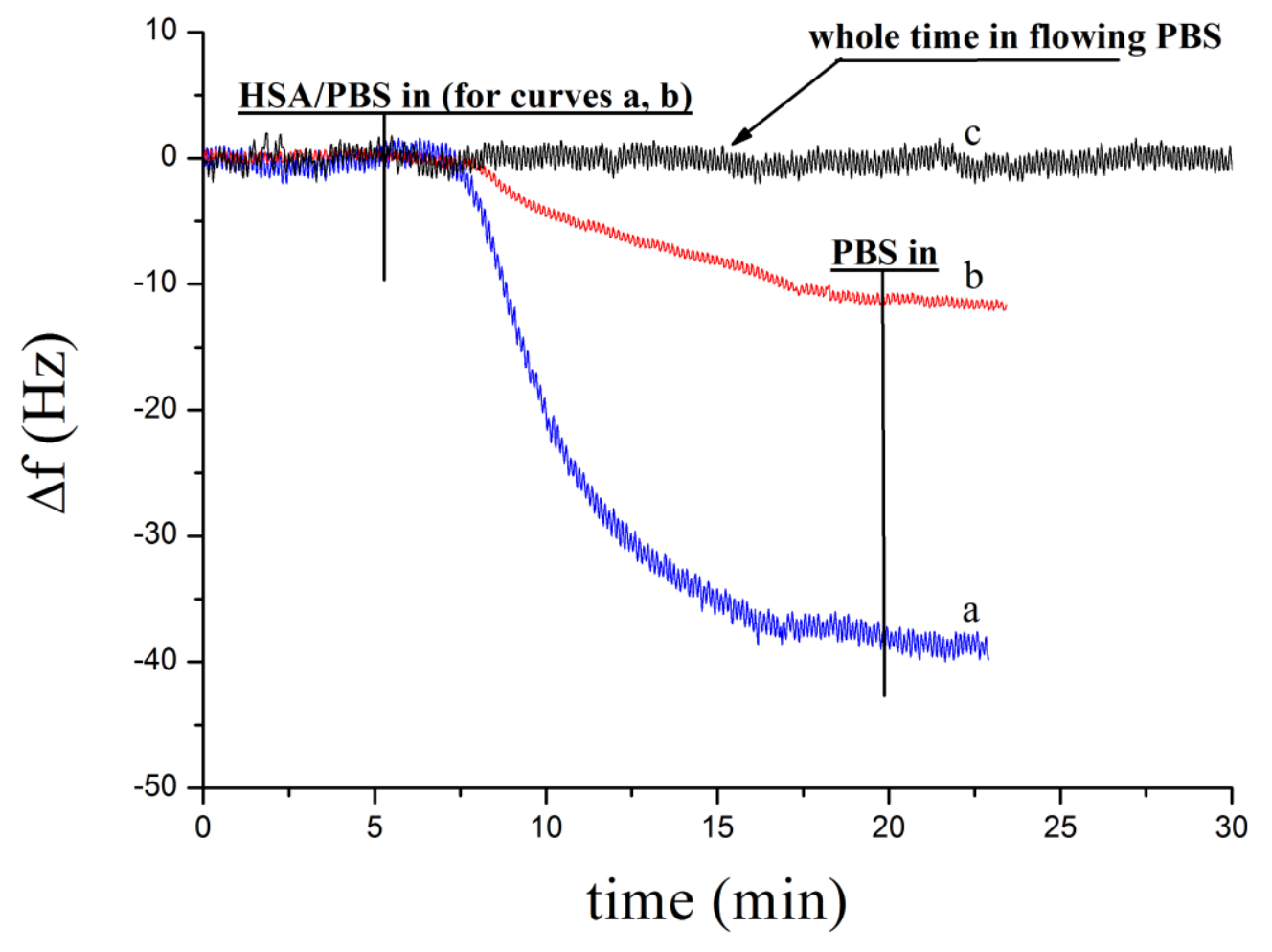




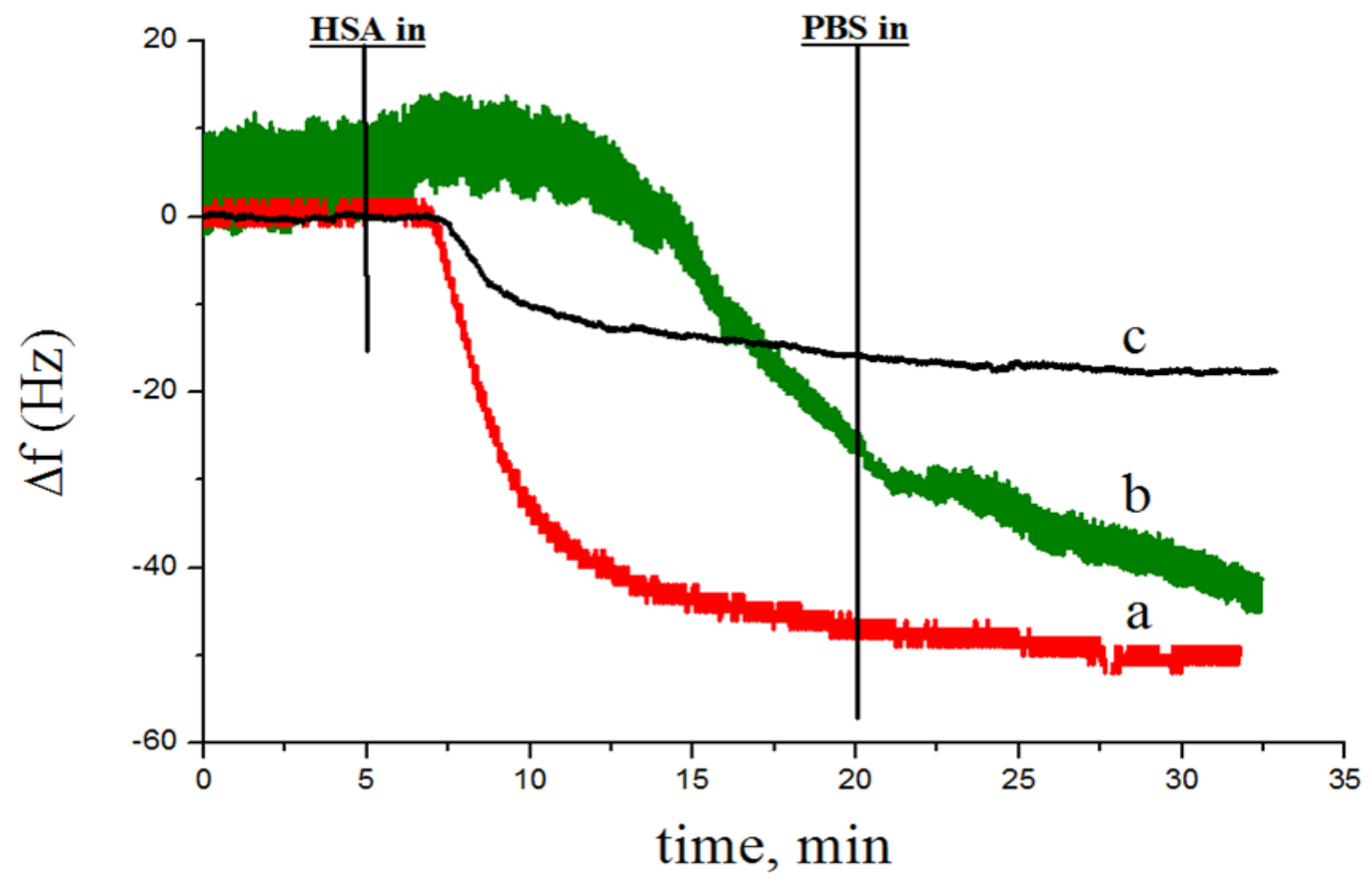




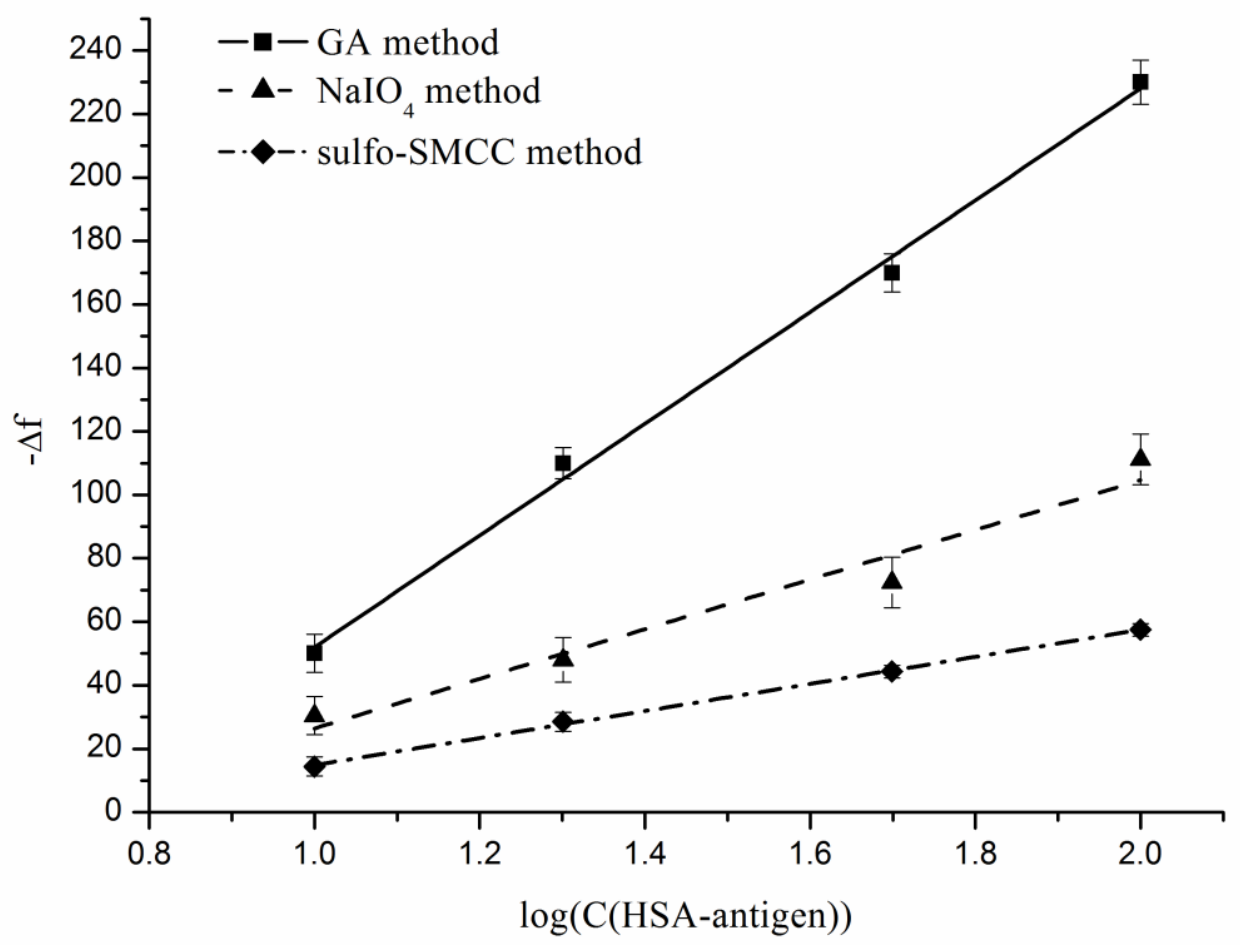

